Review

\title{
Treatment of chemotherapy-related peripheral neuropathy with traditonal Chinese medicine from the perspective of blood-arthralgia Zheng
}

\author{
Peng Cao*, Jie Yang, Xueting Cai, Xiaoning Wang, Jiege Huo* \\ Laboratory of Cellular and Molecular Biology, Jiangsu Province Institute of Traditional Chinese Medicine, Nanjing, 210028, \\ Jiangsu, China
}

\begin{abstract}
Traditional Chinese medicine classifies peripheral nerve impairment as paralysis and arthromyodynia, and considers that it is the result of defects of meridians and vessels, QI and blood, bones and muscles. Huangqi (Astragalus) Guizhi (Cassia Twig) Wuwu Tang, as a Qi invigorating formula, is usually used to improve peripheral nerve impairment. In recent years, some scholars have conducted research into Chemotherapy-induced peripheral neuropathy (CIPN) treatment with Huangqi Guizhi Wuwu Tang and certain values of this treatment approach have been identified. CIPN is a type of blood-arthralgia Zheng in traditional Chinese medicine theory. In this review, we will discuss the treatment of CIPN with Huangqi Guizhi Wuwu Tang according to blood-arthtalgia Zheng.
\end{abstract}

Keywords Chemotherapy-induced peripheral neuropathy, blood-arthtalgia, traditional Chinese medicine

\section{INTRODUCTION}

Chemotherapy-induced peripheral neuropathy (CIPN) is a common chemotherapy-related toxic and adverse effect, often found in oxaliplatin and paclitaxel, presenting sensory disturbances of the lower extremities, or the accompanied paresthesia, with or without algospasm, which is often stimulated and exacerbated under cold stimulus (Cersosimo, 2005; Chaplan et al., 1994). Some patients terminate the treatment due to the intolerant pain and numbness. Although the peripheral neurotoxicity of antineoplastic agents is not dose-limiting, its onset may severely affect the quality of life of cancer patients and cause chronic discomfort. Among the anticancer chemotherapy drugs, platinum derivates, antitubulins, thalidomide and bortezomib can induce the most severe effects on the peripheral nervous system of the treated patients (Argyriou et al., 2012). So far, preventive treatment and symptomatic treatment have not shown the exact curative effects. However, the treatment of CIPN with traditional Chinese medicine (TCM) presents satisfactory effects. In recent years, many Chinese scholars have studied this perspective for CIPN treatment. In this paper, these views/findings are summarized.

Clinical symptoms and pathogenesis of blood-arthralgia The term "Xuebi" (blood-arthralgia) first appeared in the "Nei Jing" (Internal Canon of Medicine). "Pathogenic factors enter the Yin, leading to Xuebi." (Miraculous Pivot, Nine Classical Needles). This, for the first time, points out the pathogenesis of Xuebi, yet fails to elaborate its clinical symptoms and pathogenesis. Later, Zhang Zhongjing systematically discussed Xuebi, and proposed the treatment prescription for the first time. Clinically, patients with Xuebi show that "the pulse is

*Correspondence: Peng Cao, Jiege Huo

E-mail:pcao79@yahoo.com

These authors contributed equally to this work.

Received August 9, 2012; Accepted November 5, 2012; Published

Novemver 30, 2012

doi: http://dx.doi.org/10.5667/tang.2012.0031

(C)2012 by Association of Humanitas Medicine

TANG / www.e-tang.org slightly tight at the Cunkou and Guanshang acupuncture points, a deficiency of Yin and Yang, and the pulse is slightly tight at Chizhong. The body is numb, like wandering arthritis." This indicates that the major symptom of Xuebi is "the body is numb". According to Volume Six of "Yilin Shengmo", "the numb body indicates the numbness of skin or lack of feeling of the body, just like the moment after the release from being tied by ropes". According to "General Treatise on the Cause and Symptoms of Diseases", "The numbness symptom is just like separated feelings from clothes, and the pulse at Cunkou is stable but the skin is numb." These verify that the clinical symptoms of Xuebi are the numbness of skin and limbs from feelings and temperatures. For its pathogenesis, Zhong Jing pointed out that "the rich are weak in bones and nurtured at skin, and winds lead to the disease due to their heavy perspiration". For patients who do not have a severe disease of Xuebi, acupuncture can be used to introduce Yang Qi in the body, and the patients can recover when the pulse is stable and tight. For those who have severe Xuebi, Huangqi Guizhi Wuwu Tang should be used to nurture the body and Qi (Synopsis of Golden Chamber). Later, Chao Yuanfang and Tang Rongchuan also described Xuebi in their works with similar views on its pathogenesis and treatment.

Thus, it can be inferred that the pathogenesis of blood-arthralgia can be attributed to two aspects: blood arthralgia, a disease which results in the deficiencies of Qi and blood, and the blocking of "Yang" and blood stasis due to exopathic wind. The treatment should integrate the use of needles and medicine so as to help the smooth circulation of Yang Qi in the veins. In this way, the blood supply tends to be stable and the veins will be enhanced.

\section{Clinical symptoms of CIPN and TCM understanding}

CIPN is the most prevalent neurological complication of cancer treatment, affecting a third of all patients who undergo chemotherapy. CIPN impairs functional capacities, compromises the quality of life and results in dose reduction or cessation of chemotherapy, representing a dose-limiting side effect of many antineoplastic drugs (Velasco and Bruna, 2010). 
CIPN can mainly be divided into two types: an acute, mainly cold-triggered neuropathy, occurred within hours or days after the administration of medicine, or a chronic sensory neuropathy delayed after weeks or months (multi-cycle medication) (Beijers et al., 2012). The clinical symptoms include the paresthesia or numbness of lower extremities, or atactic and motor disorders of the fine sensory. Oxaliplatin-induced CIPN also presents a remarkable feature as the enhanced numbness of distal limb at cold stimulus, which will be relieved under heating conditions (Hartmann and Lipp, 2003). Approximately $40 \%$ of the patients can recover in 6 to 8 months, but the majority of the patients will suffer for several years (Grothey et al., 2011).

According to the theory of TCM, the tumor is related with the deficiencies of Yang and Qi in the human body. As stated in "Nei Jing", "pathogenic factors gather in a body, resulting in the deficiency of Qi". Meanwhile, the toxicity of chemotherapy drugs further harms the organism, leading to the deficiency of Qi and insufficient circulation of blood. Thus, the dystrophy of limbs happens, presenting symptoms such as the numbness at the end of fingers and toes as well as hypoesthesia. CIPN is a type of qi deficiency to the blood stasis of blood-arthralgia in traditional Chinese medicine, and the pathogenesis can be attributed to the deficiencies of Qi and blood, dystrophy and health disorders, tendon dystrophy and collateral stasis. Its treatment should include tonifying Qi and nourishing blood, reconciling nutrition and health as well as promoting blood circulation and removing obstructions in channels.

Clinical research into cipn treatment with HuangQi (Astragalus) Guizhi (Cassia Twig) Wuwu Tang

Traditional Chinese medicine classifies peripheral nerve impairment as paralysis and arthromyodynia and considers that it is the result of defects of the meridians and vessels, QI and blood, bones and muscles. The Huangqi Guizhi Wuwu decoction, as a Qi invigorating formula, is usually used to improve peripheral nerve impairment (Wei et al., 2008). Some scholars have conducted research into CIPN treatment with Huangqi (Astragalus) Guizhi (Cassia Twig) Wuwu Tang and certain values of this treatment approach have been identified. Zhou et al. (2007) observed that Huangqi Guizhi Wuwu Tang presents a satisfying therapeutic effect on oxaliplatin-induced peripheral neurotoxicity and can better improve its clinical symptoms. The total efficiency rate of clinical TCM syndromes is $92.86 \%$, and the difference is statistically significant compared with the simple chemotherapy group and the Methylcobalamin group respectively $(p<0.05$ and can reduce Levi's rating $(p<0.01)$. According to EMG testing, it is found that Huangqi Guizhi Wuwu Tang can relieve the decreased conduction speed of shallow nerves led by chemotherapy drugs $(p<0.05)$. McKeage et al. (2001) evaluated the efficacy of the Jiawei Huangqi Guizhi Wuwu Decoction (JHGWD) in treating neuro-sensory toxicity induced by oxaliplatin, they found that JHGWD could prevent and reduce the occurrence and intensity of acute peripheral neuro-sensory toxicity caused by oxaliplatin. Li et al. (2006) applied Huangqi Guizhi Wuwu Tang to prevent the oxaliplatin-induced peripheral neurotoxicity. After four cycles of treatment, the statistical analysis indicated that the group with Huangqi Guizhi Wuwu Tang presented remarkable effects $(p<0.05)$. With the base of Huangqi Guizhi Wuwu Tang, it was added with earthworm, papaya, parsley, Millettia, and other medicines which help promote blood circulation and remove obstructions in channels. This approach also presented promising efficacy. The incidence of neurotoxicity in the treatment group has been significantly reduced and the degree of disease has been remarkably relieved. In addition, some scholars applied Huangqi Guizhi Wuwu Tang with the fumigation method to significantly reduce the peripheral neurotoxicity after oxaliplatin-based chemotherapy (Cheng et al., 2004).

\section{The mechanism and target of Huangqi Guizhi Wuwu Tang in the intervention of CIPN}

Currently, the occurrence mechanism of CIPN has not been clarified by all studies. Many studies indicate that dorsal root ganglion (DRG) nucleolus is the target of chronic neurotoxitocity of platinum-based drugs (Wilson et al., 2005). Oxaliplatin significantly reduced the dorsal root ganglion nucleolus diameter occurring after single doses of oxaliplatin at dose and time potins (Jamieson et al., 2003). Alterations in dorsal root ganglion nucleolus appear to be linked with the neurotoxicity of platinum-based drugs. There are studies showing that the neurotoxitocity of platinum-based drugs correlates with the rate of DRG nucleolus shrinkage during multiple-dose drug treatment (McKeage et al., 2001). Several groups have reported that the shrinkage of DRG nucleoli is associated with cisplatin (Stacchiotti et al., 1995), carboplatin (Cavaletti et al., 1998), and ormaplatin (Holmes et al., 1998). The change in the nucleolus size may come about by the inhibition of rRNA synthesis, which is a known effect of platinum-based drugs (Jordan and Carmo-Fonseca, 1998).

The intervention mechanism of traditional Chinese medicine study on CIPN is not classified deeply. In our previous study, oxaliplatin was used to build a CIPN model, and the behavioral characteristics were consistent with blood-arthralgia performance (Jamieson et al., 2003; Takeshita et al., 2011). After the treatment with Huangqi Guizhi Wuwu Tang, its mechanical pain hypersensitivity can be effectively relieved, presenting an obviously decreased paw withdrawal mechanical threshold value compared with base values and the blank group. This indicates that Huangqi Guizhi Wuwu Tang effectively relieves the numbness of limbs. Cheng (Cheng et al., 2004) and others established their models with oxaliplatin to observe the impact of Huangqi Guizhi Wuwu Tang on the conduction speeds of mouse sciatic nerves with peripheral neurotoxicity, finding that after $72 \mathrm{~h}$ of the modeling, the group with Huangqi Guizhi Wuwu Tang treatment presented faster conduction speeds and shorter incubation periods, showing significant differences $(p<0.05)$. This indicated that before the treatment with oxaliplatin, the treatment with Huangqi Guizhi Wuwu Tang can improve the conduction speed of the sciatic nerve, while shortening the lasting period of pathological changes. The complex prescription of Huangqi Guizhi Wuwu Tang promotes the in vitro survival and neurite outgrowth of DRG neurons, while enhancing their activities. Huangqi Guizhi Wuwu Tang can improve the neurotrophy and is considered to be relevant with the inhibition of the programmed death of spinal motor neurons (Tong and Hou, 2006).

\section{CONCLUSION}

The CIPN incidence mechanism has not been clarified yet. Recent studies present that the dorsal root ganglion (DRG) is a target of chronic neurotoxicity of platinum agents. As suggested by Jamieson and colleagues, one possibility is that oxaliplatin induces a decrease in phosphorylated neurofilaments in DRG neurons with a concomitant alteration in sensory axons (Jamieson et al., 2009). The mechanism of oxaliplatin-induced delayed neurotoxicity may be attributed to the participation of oxaliplatin in inhibiting the nucleolar ribosome RNA synthesis of sensory neuron bodies so that the morphology of the sensory neuron organoid becomes abnormal (Scuteri et al., 2009); chemotherapy drugs reduce the 
circulating levels of nerve growth factor in peripheral tissues, resulting in the solid contraction of the DRG neurons nucleolus and neuron protein synthesis is hindered (Cavaletti et al., 2002). Therefore, the structure and function of peripheral nerves are damaged.

In a word, no effective drug prevention and treatment with Western medicine for CIPN has been presented. According to TCM, as the clinical symptoms of blood-arthralgia shall be considered, the treatment effects of Huangqi Guizhi Wuwu Tang have been discovered in clinical practices and preliminary experiments but its mechanism of molecular biology requires further studies for clarification. As for the clinical research, the quality control and experimental design of Huangqi Guizhi Wuwu Tang also needs more stringent standards.

\section{ACKNOWLEDGEMENTS}

This work was supported by the National Natural Science Foundation of China (Nos. 81274150, 81202967 and 30873410), Natural Science Foundation of Jiangsu Province (No. SBK201241903) and Jiangsu Province's Outstanding Leader Program of Traditional Chinese Medicine.

\section{CONFLICT OF INTEREST}

The authors have declared no conflict of interest.

\section{REFERENCES}

Argyriou AA, Bruna J, Marmiroli P, Cavaletti G. Chemotherapy-induced peripheral neurotoxicity (CIPN): an update. Crit Rev Oncol Hematol. 2012;82:51-77.

Beijers AJ, Jongen JL, Vreugdenhil G. Chemotherapy-induced neurotoxicity: the value of neuroprotective strategies. Neth J Med. 2012;70:18-25.

Cavaletti G, Fabbrica D, Minoia C, Frattola L, Tredici G. Carboplatin toxic effects on the peripheral nervous system of the rat. Ann Oncol. 1998;9:443-447.

Cavaletti G, Pezzoni G, Pisano C, Oggioni N, Sala F, Zoia C, Ferrarese C, Marmiroli P, Tredici G. Cisplatin-induced peripheral neurotoxicity in rats reduces the circulating levels of nerve growth factor. Neurosci Lett. 2002;322:103-106.

Cersosimo RJ. Oxaliplatin-associated neuropathy: a review. Ann Pharmacother. 2005;39:128-135.

Chaplan SR, Bach FW, Pogrel JW, Chung JM, Yaksh TL. Quantitative assessment of tactile allodynia in the rat paw. J Neurosci Methods. 1994;53:55-63.

Cheng J, Wu G, Wu H. Clinical observation of gemcitabine and concurrent three-dimensional conformal radiotherapy (3D-CRT) in the treatment of locally advanced non-small cell lung cancer. Zhongguo Fei Ai Za Zhi. 2004;7:442-445.

Grothey A, Nikcevich DA, Sloan JA, Kugler JW, Silberstein PT, Dentchev T, Wender DB, Novotny PJ, Chitaley U, Alberts $\mathrm{SR}$, Loprinzi CL. Intravenous calcium and magnesium for oxaliplatin-induced sensory neurotoxicity in adjuvant colon cancer: NCCTG N04C7. J Clin Oncol. 2011;29:421-427.
Hartmann JT, Lipp HP. Toxicity of platinum compounds. Expert Opin Pharmacother. 2003;4:889-901.

Holmes J, Stanko J, Varchenko M, Ding H, Madden VJ, Bagnell CR, Wyrick SD, Chaney SG. Comparative neurotoxicity of oxaliplatin, cisplatin, and ormaplatin in a Wistar rat model. Toxicol Sci. 1998;46:342-351.

Jamieson SM, Liu J, Hsu T, Baguley BC, McKeage MJ. Paclitaxel induces nucleolar enlargement in dorsal root ganglion neurons in vivo reducing oxaliplatin toxicity. $\mathrm{Br} \mathrm{J}$ Cancer. 2003;88:1942-1947.

Jamieson SM, Subramaniam J, Liu JJ, Jong NN, Ip V, Connor B, McKeage MJ. Oxaliplatin-induced loss of phosphorylated heavy neurofilament subunit neuronal immunoreactivity in rat DRG tissue. Mol Pain. 2009;18:66.

Jordan P, Carmo-Fonseca M. Cisplatin inhibits synthesis of ribosomal RNA in vivo. Nucleic Acids Res. 1998;26:2831-2836.

Li Y, Cui HJ, Huang JC, Wu XQ. Clinical study of Jiawei Huangqi Guizhi Wuwu Decoction in preventing and treating peripheral neuro-sensory toxicity caused by oxaliplatin. Chin J Integr Med. 2006;12:19-23.

McKeage MJ, Hsu T, Screnci D, Haddad G, Baguley BC. Nucleolar damage correlates with neurotoxicity induced by different platinum drugs. Br J Cancer. 2001;85:1219-1225.

Scuteri A, Galimberti A, Maggioni D, Ravasi M, Pasini S, Nicolini G, Bossi M, Miloso M, Cavaletti G, Tredici G. Role of MAPKs in platinum-induced neuronal apoptosis. Neurotoxicology. 2009;30:312-319.

Stacchiotti A, Rezzani R, Rodella L, Ventura RG. Lysosomal changes in rat spinal ganglia neurons after prolonged treatment with cisplatin. Acta Anat (Basel). 1995;153:236-242.

Takeshita M, Banno Y, Nakamura M, Otsuka M, Teramachi H, Tsuchiya T, Itoch Y. The Pivotal Role of Intracellular Calcium in Oxaliplatin-Induced Inhibition of Neurite Outgrowth but Not Cell Death in Differentiated PC12 Cells. Chem Res Toxicol. 2011;24:1845-1852.

Tong Y, Hou H. Effects of Huangqi Guizhi Wuwu Tang on diabetic peripheral neuropathy. J Altern Complement Med. 2006;12:506-509.

Velasco R, Bruna J. Chemotherapy-induced peripheral neuropathy: an unresolved issue. Neurologia. 2010;25:116-131.

Wei SY, Zhang PX, Yang DM, Zhang HB, Jiang BG. [Traditional Chinese medicine and formulas of improving peripheral nerve regeneration]. Zhongguo Zhong Yao Za Zhi. 2008;33:2069-2072.

Wilson JA, Garry EM, Anderson HA, Rosie R, Colvin LA, Mitchell R, Fleetwood-Walker SM. NMDA receptor antagonist treatment at the time of nerve injury prevents injury-induced changes in spinal NR1 and NR2B subunit expression and increases the sensitivity of residual pain behaviours to subsequently administered NMDA receptor antagonists. Pain. 2005;117:421-432.

Zhou HM, Chen SQ, Sun BG, Yin LR, Ye XY, Chen ZX. A 2012 / Volume 2 / Issue 4 / e30 
Treatment of CIPN with TCM from blood-arthralgia Zheng

clinical research of Jiawei simiaosan oral administration combined with the external application of Sihuangshuimi in the treatment of acute gouty arthritis. Zhong Yao Cai. 2007;30:1196-1198. 